\title{
Cocaína y escroto agudo ¿Existe alguna relación?
}

\section{Cocaine and acute scrotum: Is there an association?}

\author{
Guillermo Patricio Campos-Márquez, ${ }^{1}$ Jorge Eduardo Espino-Galván, ${ }^{2}$ Iris Natalia Cruz-Zamudio, ${ }^{2}$ Mario \\ Vásquez-Hernández ${ }^{2}$
}

\begin{abstract}
Resumen
ANTECEDENTES: El efecto de la cocaína en los testículos provoca un proceso isquémico, debido al bloqueo de la recaptación de neurotransmisores, con subsiguiente vasoconstricción local. Es probable que la cocaína genere episodios repetidos de isquemia del cordón testicular, daño vascular crónico, incluso un cuadro de escroto agudo con afectación vascular aguda irreversible.

CASO CLínICO: Paciente masculino de 58 años, que acudió al servicio de Urgencias por dolor súbito en la región testicular izquierda, posterior a la inhalación de cocaína. Entre sus antecedentes personales refirió dependencia a la cocaína desde los 20 años de edad; antecedentes médicos: hipertensión arterial sistémica diagnosticada un año antes, sin tratamiento actual. En la exploración urológica se observó: paciente en bipedestación con eritema testicular, escroto edematizado, testículo ascendido y horizontal; doloroso a la palpación, epidídimo en posición anterior y aumentado de volumen; reflejo cremasteriano abolido, sin mancha testicular azulada. El ultrasonido evidenció: testículo izquierdo de $4 \mathrm{~cm}$, ecogenicidad heterogénea, epidídimo engrosado y fibroso (3.5 cm de espesor), con múltiples adherencias en el testículo y en la pared escrotal, e hidrocele de 40 cc, aproximadamente. Según los criterios de la escala de TWIST se clasificó al pacientes en riesgo alto, por lo que se decidió efectuar exploración quirúrgica inmediata, donde se observó el cordón testicular no viable. El tratamiento definitivo consistió en orquiectomía izquierda, con evolución satisfactoria. CONCLUSIÓN: Es importante investigar el antecedente de consumo de cocaína en pacientes con síndrome metabólico, pues representa un factor decisivo en el inicio del cuadro clínico de escroto agudo.
\end{abstract}

PALABRAS CLAVE: Escroto agudo; consumo de cocaína; síndrome metabólico.

\section{Abstract}

BACKGROUND: The effect of cocaine at the testicular level is indirectly due to an ischemic process resulting from the blocking of neurotransmitter reuptake, which leads to local vasoconstriction. Cocaine users may be prone to present with repeated episodes of spermatic cord ischemia, causing chronic vascular damage. Symptoms of acute scrotum can result in irreversible acute vascular damage.

CLINICAL CASE: A 58-year-old man arrived at the emergency service due to pain of sudden onset at the left testicular region after having inhaled cocaine. He had a personal history of cocaine addiction since the age of 20 years and a medical history of high blood pressure of one-year progression, with no current treatment. Urologic examination: with the patient standing on both feet, testicular erythema, scrotum with edema, and ascended testis with a horizontal lie were observed. Palpation was painful, the epididymis was enlarged and in the anterior position, and there was no cremasteric reflex or testicular "blue dot" sign. Ultrasound study: left testis measuring $4 \mathrm{~cm}$, with heterogeneous echogenicity, a fibrous thickened epididymis (thickening of $3.5 \mathrm{~cm}$ ) with numerous testicular and scrotal wall adhesions, and hydrocele of approximately $40 \mathrm{cc}$. The patient was classified as high-risk on the TWIST scale and so immediate surgical examination was carried out, revealing a non-viable spermatic cord. Left orchiectomy was performed as definitive treatment, and postoperative progression was satisfactory. CONCLUSION: The evaluation of a history of cocaine use in patients with metabolic syndrome is important, given that it can be a decisive factor in the appearance of acute scrotum.

KEYWORDS: Acute scrotum; Cocaine use; Metabolic syndrome.

\footnotetext{
${ }^{1}$ Departamento de Urología.

2Departamento de Cirugía general.
}

Hospital General de Matamoros Dr. Alfredo Pumarejo, Tamaulipas, México.

Recibido: agosto 2018

Aceptado: octubre 2018

Correspondencia Guillermo Patricio Campos Márquez dr.patricio.c.m@gmail.com Facebook: Patricio $\mathrm{Cm}$

Este artículo debe citarse como Campos-Márquez G, Espino-Galván JE, Cruz-Zamudio IN, Vásquez-Hernández M. Cocaína y escroto agudo ¿Existe alguna relación? Rev Mex Urol. 2018 nov-dic;78(6):452-57.

DOI: https://doi.org/10.24245/revmexurol.v78i6.2489 


\section{ANTECEDENTES}

La cocaína es un alcaloide natural, extraído de las hojas de Erythroxylum coca, que se metaboliza por las esterasas hepáticas y plasmáticas a sustancias activas e inactivas, para finalmente excretarse por la orina. El inicio y la duración de los efectos de la cocaína es rápido y breve, respectivamente. Debido a la intensa vascularización de la mucosa nasofaríngea, la absorción es rápida, así como sus efectos. El pico plasmático se produce con intervalos de 30 a 120 minutos después de la administración nasal u oral; los límites mínimo y máximo de las dosis de cocaína varían de 0.2 a 3-4 mg/kg y las concentraciones plasmáticas máximas ocurren de 50 a 2000 ng/ $\mathrm{mL}$. Esta sustancia potencia los efectos simpáticos agudos sobre el sistema cardiovascular, con aumento del efecto inotrópico y cronotrópico, e incremento de la vasoconstricción periférica, incluso de los efectos trombogénicos secundarios. El efecto vasoconstrictor de la cocaína se debe, principalmente, a la estimulación de los receptores $\alpha$-adrenérgicos en las células del músculo liso arterial, además del aumento de la concentración de endotelina-1 y disminución de las concentraciones sanguíneas de óxido nítrico. La cocaína inhibe la recaptación presináptica de aminas biogénicas como: norepinefrina, dopamina y serotonina en el organismo, con subsiguientes efectos sistémicos que provocan vasoconstricción difusa. Los efectos trombogénicos de la cocaína se atribuyen al aumento en la actividad del inhibidor de plasminógeno-activado, además de la concentración, activación y agregación plaquetaria. La cocaína puede afectar el sistema vascular en forma aguda y crónica. El daño agudo se centra en la pérdida de la función protectora del endotelio, que se considera un denominador común en la patogenia de la enfermedad vascular isquémica. Los efectos crónicos consisten en daño endotelial repetido, que resulta en formación prematura de aterosclerosis severa. ${ }^{1-4}$
La acción de la cocaína en la circulación testicular se ha reportado muy poco en la bibliografía. Li y sus colaboradores estudiaron el efecto de la cocaína en la circulación testicular en ratas y encontraron que la isquemia ocurre por acción indirecta, al bloquear la recaptación de neurotransmisores, que resulta en vasoconstricción local. Yang y colegas informaron que la cocaína en ratas provocó vasoconstricción crónica y daño testicular. Estos hallazgos pueden sugerir cuadros clínicos de escroto agudo, debido al alto grado de isquemia en el cordón testicular, y su único tratamiento consistirá en cirugía..$^{5-7}$

Los pacientes con escroto agudo requieren pronta evaluación; existen múltiples diagnósticos diferenciales relacionados con esta alteración y debido a la superposición de síntomas y signos, es difícil indicar tratamiento quirúrgico. Barbosa y sus coautores reportaron la escala TWIST (por sus siglas en inglés: Testicular Workup for Ischemia and Suspected Torsion), basada en cinco parámetros clínicos: hinchazón testicular (2 puntos), testículo duro (2 puntos), ausencia de reflejo cremastérico (1 punto), náuseas-vómitos (1 punto) y testículos ascendidos (1 punto). Los pacientes se dividen en grupos de riesgo: bajo (0-2 puntos), intermedio (3-4 puntos) y alto (5-7 puntos), con la intención de decidir qué pacientes requerirán exploración testicular quirúrgica. Esta técnica supone valores predictivos negativo y positivo de $100 \%$ para los grupos de bajo y alto riesgo, respectivamente. ${ }^{8}$

Algunos estudios señalan como probable factor desencadenante de escroto agudo el consumo de cocaína en pacientes mayores de 35 años, puesto que la torsión testicular es la causa principal en solo $6 \%$ de los pacientes de ese grupo etario. ${ }^{9}$

\section{CASO CLÍNICO}

Paciente de 58 años, que acudió al servicio de Urgencias del Hospital General de Matamoros Dr. Alfredo Pumarejo por dolor súbito punzante 
continuo y aumento de volumen en la región testicular izquierda, que no disminuía en ninguna posición e impedía la deambulación. El paciente refirió haber inhalado cocaína cuatro días antes de su ingreso a la unidad médica. Debido a sus síntomas se autoprescribió analgésicos e inhaló aproximadamente $30 \mathrm{mg}$ /día de cocaína sin obtener mejoría. Incluso, señaló haber sufrido un cuadro similar 3 meses antes, después de inhalar cocaína, con disminución del dolor mediante el consumo de analgésicos no especificados.

Entre sus antecedentes personales refirió dependencia a la cocaína desde los 20 años de edad (consumo de 30-60 mg cada quince días, en su hogar) y abuso de alcohol. Antecedentes médicos de hipertensión arterial sistémica diagnosticada un año antes, sin tratamiento actual, y como antecedentes sexuales: inicio de vida sexual activa a los 17 años, heterosexual, tres parejas en el último año con utilización de preservativo.

A la exploración física se encontró: pulso 100 latidos/min; frecuencia respiratoria 20 respiraciones por minuto; tensión arterial 140/80 $\mathrm{mmHg}$; peso $94 \mathrm{~kg}$ y talla $170 \mathrm{~cm}$. El paciente se encontraba consciente, orientado, fascies de preocupación, mucosas con moderada hidratación, palidez de tegumentos (++). Exploración urológica: paciente en bipedestación con eritema testicular, escroto edematizado, testículo ascendido y horizontal; doloroso a la palpación, epidídimo en posición anterior y aumentado de volumen; reflejo cremasteriano abolido, sin mancha testicular azulada.

Los estudios de laboratorio reportaron: biometría hemática con leucocitos $21.9 \times 10^{3} \mathrm{UL}$, neutrófilos $74.5 \%$; hemoglobina $14.1 \mathrm{~g} / \mathrm{dL}$, hematocrito 41.3 y plaquetas $336 \times 10^{3}$ UL. Química sanguínea: glucosa $104 \mathrm{mg} / \mathrm{dL}$, urea $26 \mathrm{mg} / \mathrm{dL}$, creatinina $0.9 \mathrm{mg} / \mathrm{dL}$, ácido úrico $5.3 \mathrm{mg} / \mathrm{dL}$, colesterol $225 \mathrm{mg} / \mathrm{dL}$, triglicéridos $156 \mathrm{mg} / \mathrm{dL}$, HDL 36 mg/dL, VLDL 45 mg/dL, albúmina 4 g/
dL. Examen general de orina: color amarillo de aspecto claro; densidad 1.015, pH 6; glucosa, proteínas, hemoglobina, cuerpos cetónicos, pigmentos biliares y nitritos negativos; leucocitos 0-1 por campo; bacterias y levaduras negativas, y eritrocitos de 0 por campo; prueba de cocaína en orina positiva.

El ultrasonido evidenció: testículo izquierdo de $4 \mathrm{~cm}$, ecogenicidad heterogénea, epidídimo engrosado y fibroso $(3.5 \mathrm{~cm}$ de espesor), con múltiples adherencias en el testículo y en la pared escrotal, e hidrocele de 40 cc aproximadamente. Se estableció el diagnóstico sugerente de torsión testicular. Figura 1

Se decidió la exploración quirúrgica inmediata, debido al alto riesgo en la escala TWIST: criterios de inflamación testicular (2 puntos), testículo duro (2 puntos), ausencia de reflejo cremastérico (1 punto), náuseas-vómitos (1 punto) y testículos ascendidos (1 punto), con sumatoria de 7 puntos.

En el transquirúrgico se encontró: cordón testicular sin datos de torsión franca, hallazgos sugerentes de afectación vascular grave y cambios isquémicos y necróticos en la arquitectura testicular (Figura 2). Como medida inicial se colocaron compresas con solución salina tibia sobre el área afectada durante 15 minutos, sin obtener reacción satisfactoria; por tanto, se decidió efectuar orquiectomía izquierda. La evolución posoperatoria transcurrió sin incidencias, por lo que se otorgó el alta hospitalaria.

El estudio anatomopatológico reportó, como hallazgos macroscópicos: isquemia tubular, congestión intraparenquimatosa severa, y congestión vascular severa del cordón espermático, compatible con obstrucción proximal vascular. Entre los hallazgos microscópicos se observó: fibrosis intersticial con parénquima testicular, ausencia de células germinales en los túbulos seminíferos y abundantes neutrófilos en la luz tubular. Figura 3 

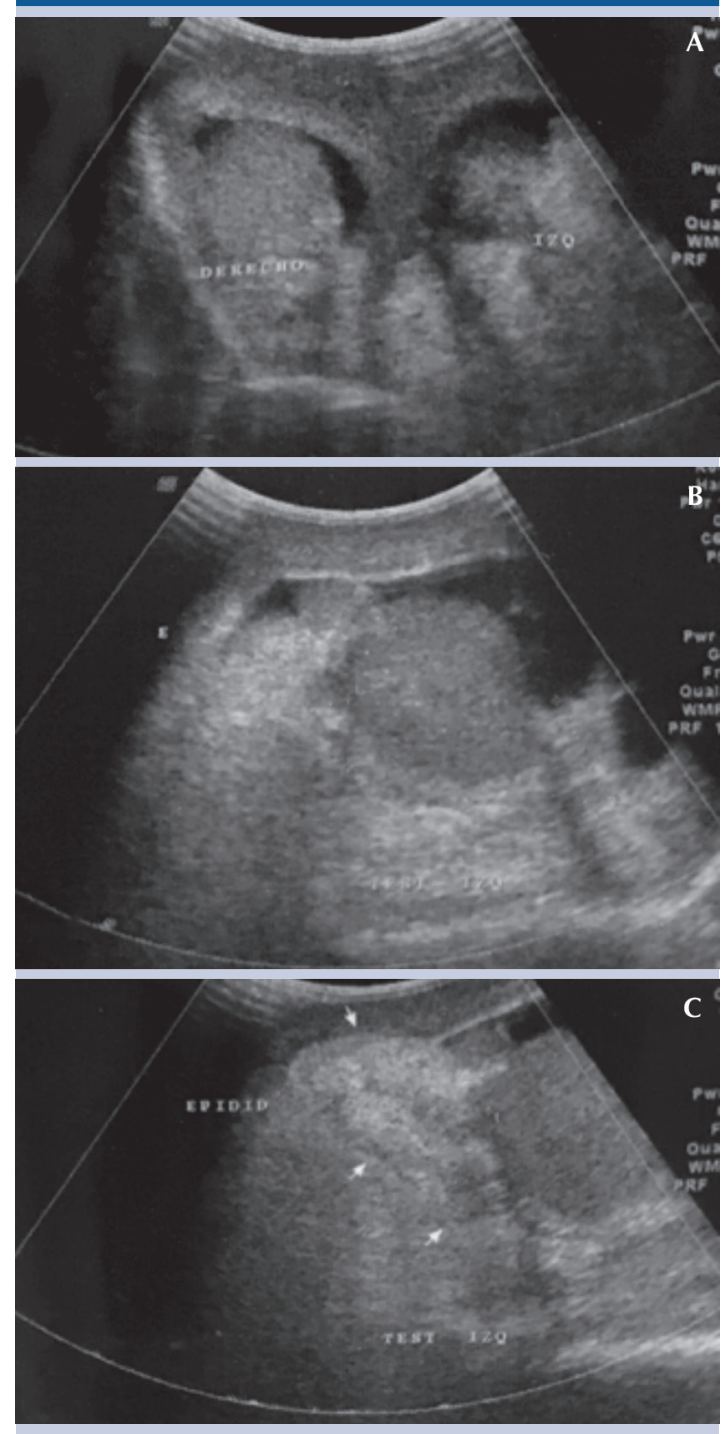

Figura 1. Ultrasonido testicular. A) Testículo izquierdo de $4 \mathrm{~cm}$, con ecogenicidad heterogénea, B) hidrocele de 40 cc aproximadamente, C) epidídimo engrosado fibroso (3.5 cm de espesor), con múltiples adherencias al testículo y a la pared escrotal.

\section{DISCUSIÓN}

Del caso aquí expuesto destaca la importancia de investigar el antecedente de consumo de cocaína como factor desencadenante de escroto

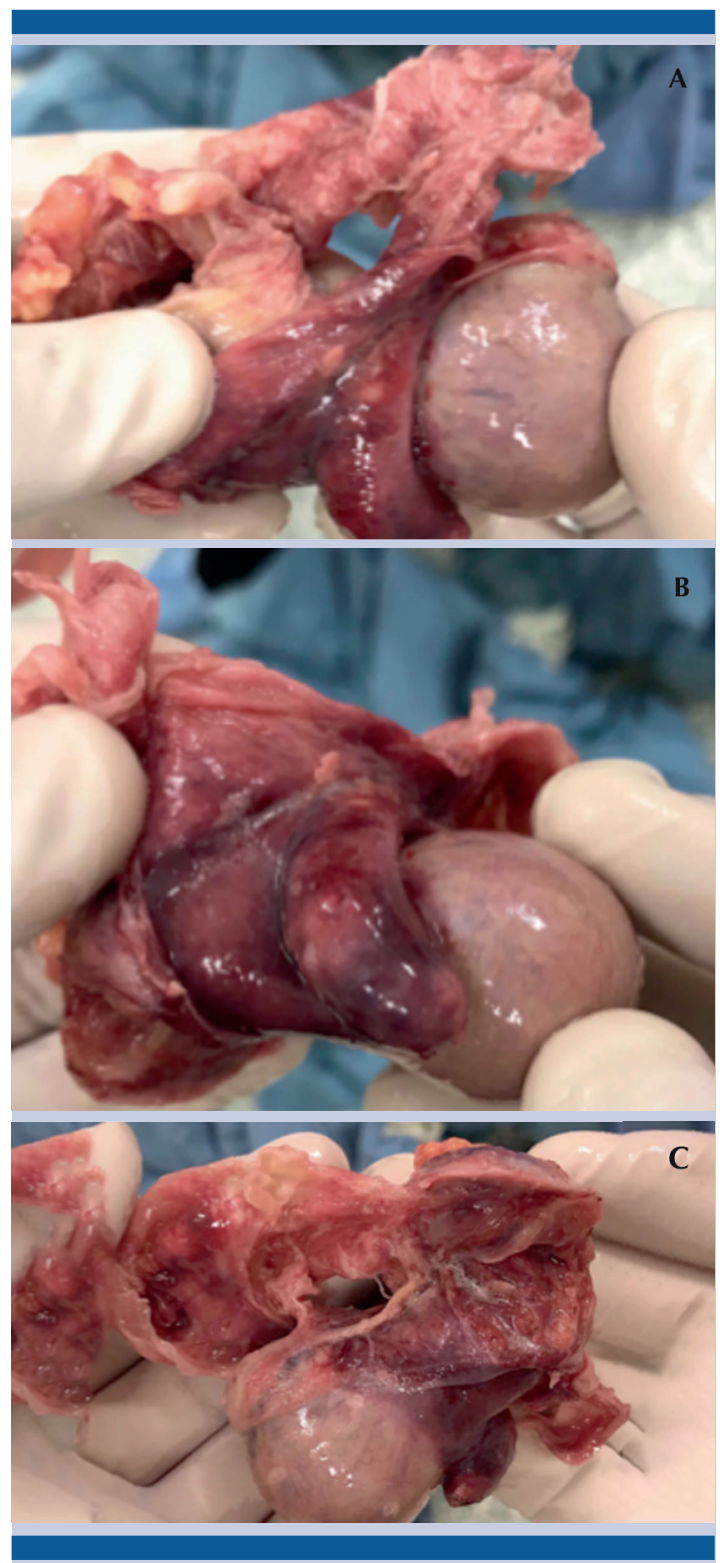

Figura 2. Pieza quirúrgica: orquiectomía izquierda. A) Cordón testicular sin torsión franca, B) necrosis isquémica en área triangular, bien delimitada, del cordón testicular, y C) parénquima normal sustituido por tejido fibroso.

agudo, con la intención de establecer los protocolos de prevención, diagnóstico y tratamiento oportunos. Si bien el uso de esta substancia es poco documentada, es importante considerarla 


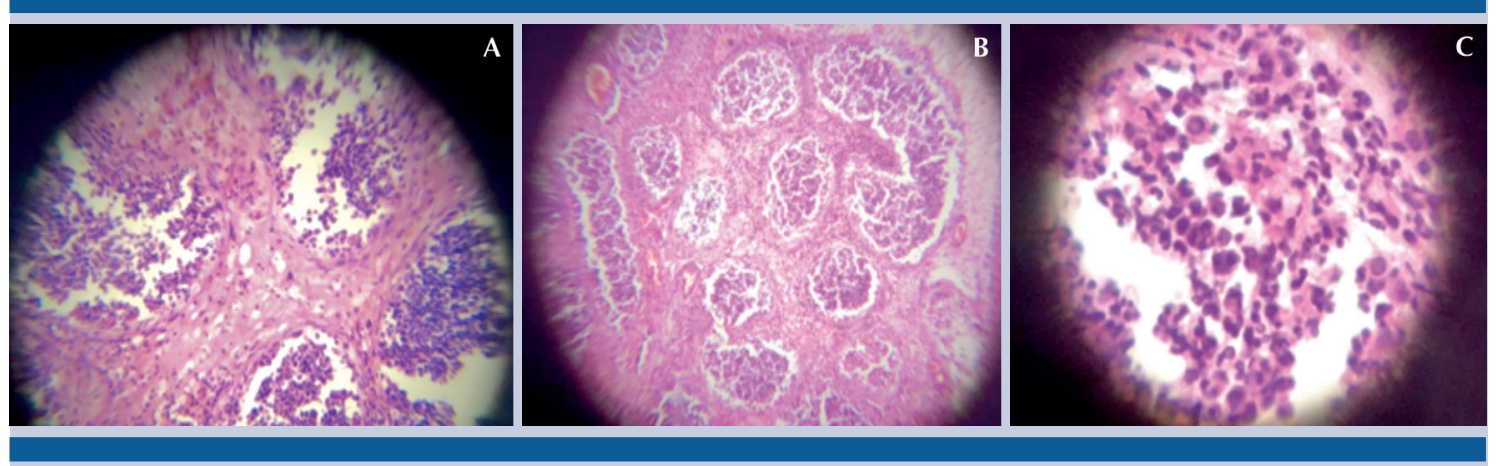

Figura 3. Corte microscópico. A) Fibrosis intersticial, B) ausencia de células germinales en los túbulos seminíferos, y C) abundantes neutrófilos en la luz tubular.

en pacientes con alteraciones testiculares de este tipo, porque sigue siendo un reto diagnóstico.

El ultrasonido Doppler es un estudio con excelente sensibilidad diagnóstica, pues evidencia el flujo vascular deficiente del testículo afectado, como sucedió en el paciente de este ensayo. Para evaluar la estructura escrotal, la ecografía es un estudio que depende del tiempo de evolución de la enfermedad, pues la escala de grises puede resultar normal en las primeras 4-6 horas del evento isquémico del cordón espermático. Mientras avanza el proceso inflamatorio disminuye la señal ecoica y al cabo de 24 horas existe mayor heterogeneidad (esta última relacionada con deficiente preservación o rescate testicular). ${ }^{10}$

En este caso clínico se demuestra el poco conocimiento del consumo de cocaína como factor desencadenante de escroto agudo; sin embargo, con los criterios de TWIST pueden identificarse los pacientes que ameritan exploración quirúrgica urgente y lograr la preservación del testículo. En este estudio, el tiempo de evolución del cuadro fue mayor a 96 horas, por lo que no fue posible revertir el daño del cordón espermático.

El tratamiento de pacientes con cuadro clínico de escroto agudo, en gran porcentaje, consiste en exploración quirúrgica urgente. El caso reportado en este estudio no tuvo datos de torsión testicular evidente; sin embargo, se observó isquemia generalizada en todo el cordón espermático, con zonas de necrosis, que después de realizar las medidas de salvamento no se logró recuperar la viabilidad del testículo, por lo que se decidió efectuar orquiectomía izquierda.

El estudio histopatológico comprobó el daño endotelial, además de elevada concentración de neutrófilos (proceso agudo) y fibrosis intersticial (proceso crónico).

El paciente de este ensayo fue diagnosticado con síndrome metabólico. La alteración de la función de las LDL ocurre porque la íntima está flanqueada por dos barreras permeables (el endotelio y la túnica media). Además, la ausencia de vasos linfáticos aumenta la permanencia de las LDL en ese espacio, lo que implica su fácil degradación y oxidación. Los macrófagos pueden sobrepasar su capacidad fagocítica debido al flujo masivo de las LDL degradadas. La hipertensión provoca engrosamiento en la íntima y la capa media, lo que resulta en aterosclerosis. Diversos estudios señalan que la mayor parte de los factores de riesgo cardiovascular se relacionan con inflamación 
crónica. Incluso se ha propuesto la leucocitosis como marcador inespecífico de inflamación crónica, en la evolución a estados protrombóticos y efectos vasculares nocivos como: incremento de radicales superóxido y mieloperoxidasa, y aceleración de la aterosclerosis debida a la elevada oxidación de partículas de LDL y HDL, que en conjunto con la cocaína pueden considerarse factores desencadenantes de daño vascular crónico y agudo del cordón testicular. ${ }^{11}$

\section{CONCLUSIÓN}

El escroto agudo no representa un cuadro clínico específico de adolescentes, incluso los adultos tienen mayor riesgo de padecerlo; por tanto, es necesario realizar un adecuado interrogatorio, con la intención de identificar los factores predisponentes. Aunque existen diversos datos relacionados con la disfunción endotelial inducida por la cocaína de forma aguda y crónica a nivel vascular, la relación entre el consumo de cocaína e isquemia del cordón testicular, además de síndrome metabólico, no se encuentran del todo descritos. Es importante evaluar los efectos vasculares testiculares en pacientes con antecedente de consumo de cocaína, para de esta forma lograr una adecuada prevención y establecer el tratamiento oportuno.

\section{REFERENCIAS}

1. Bachi, et al. Vascular disease in cocaine addiction. Atherosclerosis 2017;262:154-162. http://dx.doi.org/10.1016/j. atherosclerosis.2017.03.019

2. Havakuk O, et al. The Cardiovascular effects of cocaine. J Am Coll Cardiol 2017;70:101-13. DOI: 10.101d6/j. jacc.2017.05.014

3. Zimmeddrman JL. Cocaine intoxication. Crit Care Clin 2012;28(4):517-526. DOI: 10.1016/j.ccc.2012.07.003

4. Lorenzo P, et al. Cocaína: aspectos farmacológicos. Adicciones 2001;13:179-190. Dirección URL: < https://medes.com/ publication/4970>.

5. Tamanini JT, et al. Cocaine abuse that presents with acute scrotal pain and mimics testicular torsion. Int Braz J Urol 2016;42(5):1028-1032. DOI: 10.1590/S1677-5538. IBJU.2015.0663

6. Li H, et al. Cocaine induced apoptosis in rat testes. J Urol 1999;162:213-216. DOI: 10.1097/00005392-19990700000070

7. Yang GD, et al. Effect of cocaine on germ cell apoptosis in rats at different ages. Asian J Androl 2006;8(5):569-575.

8. Manohar CS, et al. Evaluation of Testicular Workup for Ischemia and Suspected Torsion score in patients presenting with acute scrotum. Urol Ann 2018;10:20-3. DOI: 10.1111/j.17457262.2006.00191.x DOI: c10.4103/UA.UA_35_17a

9. Tang YH, et al. A 55-Year-Old Man with Right Testicular Pain: Too Old for Torsion? Urology Case Reports. 2017; 11:74-75. DOI: 10.1016/j.eucr.2016.11.023

10. McDowall, et al. The ultrasonographic "whirlpool sign" in testicular torsion: valuable tool or waste of valuable time? A systematic review and meta-analysis. Emerg Radiol. 2018;25: 281-292. DOI: 10.1007/s10140-018-1579-x

11. Laclaustra-Gimeno, et al. Síndrome metabólico. Concepto y fisiopatología. Rev Esp Cardiol 2005;5(D):3D-10D. Dirección URL: <http://www.revespcardiol.org/es/content/ articulo/13083442/>. 